\title{
Apropos of Signal Processing
}

\author{
Asoke K. Nandi * \\ Department of Electronic and Electrical Engineering, Brunel University London, Uxbridge, United Kingdom
}

Keywords: signal processing, artificial intelligence, academic impacts, societal impacts, global challenges

Many of the problems addressed by signal processing can be classified as detection, estimation, or prediction. All humans do signal processing every day. For example, before crossing a road we detect cars; if there is a car, we estimate how far away the car is and predict if we will be able to cross the road before the car arrives. In fact, signal processing is not only essential for humans but also for all animals and plants. Perhaps signal processing is a fundamental requirement for the existence of life.

The research field of signal processing is a comparatively new field. There are both analogue signal processing and digital signal processing. Digital Signal Processing is some 60 years old, but some aspects of it can be traced back to mathematics as early as 18th century. In general, the problems of detection, estimation, and prediction in the real world will never be solved with $100 \%$ accuracy. Thus, these will remain with us forever. Even if a particular problem is solved with $100 \%$ accuracy, one can look for improvements in the form of reduced computational complexity or reduced number of samples or lower signal-to-noise ratio, etc. So, signal processing is here to stay. One of the many encouraging aspects is that the signal processing community continues to find newer applications in different areas. The prognosis is that signal processing will continue to flourish much as it has been doing in communications signal processing, biomedical signal processing, genomic signal processing, brain signal processing, etc. The future of signal processing is bright.

One way to map out signal processing is as art, algorithms, and architecture. Here art refers to theoretical ideas. Our community has been very successful in adopting and adapting existing theoretical ideas as well as developing new ones, e.g., independent component analysis and compressive sampling. An algorithm refers to an implementation of theory to solve a specific problem. Again, we have been very successful in this. Although an algorithm refers to a software implementation, it needs to be implemented on hardware to produce results. There has been success with both specific and generic hardware. Of course, our progress has been helped by the rapidly increasing speed, memory, and other developments of integrated circuits as well as our entrepreneurial spirit of building on what is available or possible. Combinations of signal processing and artificial intelligence have been opening up new frontiers. In recent decades many more opportunities for interdisciplinary research have emerged; we should continue to embrace them and to look for new ones. In the comparatively short history of the signal processing research field, the breadth and depth of our contributions in biomedicine, communications, genomics, seismology, digital cameras, mobile phones, and many others are amazing. The huge success of signal processing in such a comparatively short time is a testament to the hard work and vision of not only this generation but also previous generations of signal processors.

On a personal note, it is an honour to have been invited to become the inaugural Field Chief Editor of the Frontiers in Signal Processing journal (https://www.frontiersin.org/journals/signal-processing). As it is a new journal it will provide an additional opportunity for the community to publish manuscripts in a new journal and at the same time it will have to compete with other journals covering this subject area. It is hoped and believed that the competition would help drive up standards, as well as timeliness and accessibility of publications, without compromising quality. As this is a completely open-access journal, all the published manuscripts will be available for studying by anybody in the world, independent of her/his social, cultural, and economic circumstances, so long as s/he has access to the internet and a "computer." Therefore, by publishing open access, the research will be more readily available not only to other researchers, but also to the people who will 
ultimately benefit most from the applications of Signal Processing (e.g., non-academic or private sector entrepreneurs, governmental bodies, and even the general public). Inevitably, this will serve as an outreach activity of this journal and inform people of the very latest research and results.

The Frontiers in Signal Processing journal has been launched recently with five sections - Image Processing, Radar Signal Processing, Signal Processing for Communications, Signal Processing Theory, and Statistical Signal Processing. These sections are led by well-known and active researchers, and they are supported by many associate editors and reviewers as well as the journal staff. This augurs well for the review process of manuscripts as well as the timeliness and the quality of the publications. Gradually, other sections will be added to the Frontiers in Signal Processing journal.

Each of these five sections will publish Grand Challenges relevant to the particular section in the near future. Undoubtedly many technical challenges will be solved and many inventions will be made in the years to come, and they will be published in Frontiers in Signal Processing journal. In the process we need to consider both academic and societal impacts of our research. Generally, the academic impacts are the better addressed of the two by informing other researchers in our research community and influencing them. Often these impacts are measured by, amongst other things, the number of citations, $\mathrm{H}$-index, etc. Interdisciplinary research has been growing significantly and it is expected to do so for the foreseeable future. So, we need to inform and influence researchers in other communities more. To help achieve this, there is a mechanism in the Frontiers framework through "cross-listing" (see "Journal Structure" here: https://www.frontiersin.org/about/publishingmodel). This allows research published in one Frontiers journal to be visible also on another Frontiers journal's website and, in so doing, promotes interdisciplinary discussions and facilitates wider applications.

As well as academic impacts, societal impacts are even more important to consider. Whilst it is true that we live in a society which is part of a nation, in reality we are inherently and deeply connected to the whole world in a way that has never been the case before the year 1990 . This has been primarily possible by the significant improvements in telecommunications and transportations, as well as the invention of the internet and widespread availability of laptops and mobile phones. In this highly connected world, a problem in one country may become a bigger problem in another country and indeed the whole world very quickly; computer security breach, polluted environments, and Covid-19 are three good examples.
There are many global challenges, e.g., environment, energy, assisted living, poverty, equality of opportunities, etc. As well as paying attentions to problems here and now, it is important to spend some time and effort to address things for the future - ours and beyond. Of course, it is pleasurable to do something and see the positive results immediately (e.g., going to a restaurant and enjoying a meal), but it is also pleasurable to plant a rose bush and see it flower several months or a year later. Furthermore, going back several hundred years, it typically took 50 years for the roof of a cathedral to settle from its starting position. This meant that people who designed the roof and laid the roof did not live to enjoy the final outcome of their own work (by the way, they did detect, estimate, predict, and use transferable learning), yet other people have been admiring such a roof even hundreds of years later. It would be good to do some things, like addressing global challenges, for posterity.

Thinking more about societal impacts, our community needs to evince the achievements of signal processing and its emerging capabilities to policy makers to help them make informed decisions. It is important to explain to our existing and potential funders of how our abilities and achievements align with their plans and help develop better plans. It would be helpful to outline to the general public some of our achievements and capabilities that relate to their lives. For example, if we were to take out everything that relies on signal processing, the world would be unrecognisable compared to the one we inhabit. As well as effective communication of our methods and results, there is a need to champion values, because values are enduring and often values capture people's imagination. At the core of all these, we must remember the importance of educating young people and of enthusing and engaging them in our signal processing community as they will determine the future of our field that we all love and work for.

\section{AUTHOR CONTRIBUTIONS}

The author (AN) conceived and wrote the entire document. He has shown it to five people for their comments.

Conflict of Interest: The author declares that the research was conducted in the absence of any commercial or financial relationships that could be construed as a potential conflict of interest.

Copyright (c) 2021 Nandi. This is an open-access article distributed under the terms of the Creative Commons Attribution License (CC BY). The use, distribution or reproduction in other forums is permitted, provided the original author(s) and the copyright owner(s) are credited and that the original publication in this journal is cited, in accordance with accepted academic practice. No use, distribution or reproduction is permitted which does not comply with these terms. 